\title{
Empfehlungen zur Durchführung einer Bronchoskopie in Zeiten der COVID-19-Pandemie: Update 12/2020
}

\section{Recommendations for Performing Bronchoscopy in Times of the COVID-19 Pandemic - Update 12/2020}

Autoren

K. Darwiche1, B. Ross², W. Gesierich ${ }^{3}$, C. Petermann ${ }^{4}$, R.-H. Huebner ${ }^{5}$, C. Grah ${ }^{6}$, D. Gompelmann ${ }^{7}$, J. Hetzel ${ }^{8,9}$, A. Holland ${ }^{10}$, S. Eisenmann ${ }^{11}$, F. Stanzel ${ }^{12}$, A. Kempa ${ }^{13}$, K. Conrad ${ }^{14}$, L. Hagmeyer ${ }^{15}$, R. Eberhardt ${ }^{16,17}$

Institute

1 Klinik für Pneumologie, Sektion Interventionelle Bronchologie, Ruhrlandklinik - Universitätsmedizin Essen

2 Krankenhaushygiene, Universitätsmedizin Essen

3 Klinik für Pneumologie, Asklepios Fachkliniken München-Gauting

4 Klinik für Pneumologie, Asklepios Klinikum Harburg, Hamburg

5 Medizinische Klinik mit Schwerpunkt Infektiologie und Pneumologie, Charité - Universitätsmedizin Berlin

6 Klinik für Innere Medizin, Schwerpunkt Pneumologie und Lungenkrebszentrum (DKG),

Gemeinschaftskrankenhaus Havelhöhe, Berlin

7 Klinische Abteilung für Pulmologie, Universitätsklinik für Innere Medizin II, Medizinische Universität Wien, Österreich

8 Klinik für medizinische Onkologie und Pneumologie, Universitätsklinikum Tübingen

9 Klinik für Innere Medizin, Abteilung Pneumologie, Kantonsspital Winterthur, Schweiz

10 Klinik für Innere Medizin SP Pneumologie, Universitätsklinikum Gießen und Marburg

11 Klinik für Innere Medizin I, Universitätsklinikum Halle (Saale)

12 Pneumologie - Thorakale Endoskopie, Lungenklinik Hemer

13 Pneumologie, Medizinische Klinik I, Klinik Löwenstein

14 Pneumologie, Lungenklinik Ballenstedt/Harz

15 Klinik für Pneumologie und Allergologie, Zentrum für Schlaf- und Beatmungsmedizin, Krankenhaus Bethanien, Solingen

16 Pneumologie und Beatmungsmedizin, Thoraxklinik am Universitätsklinikum Heidelberg

17 Translational Lung Research Center Heidelberg (TLRCH), Deutsches Zentrum für Lungenforschung (DZL)

online publiziert $\quad 11.12 .2020$
Bibliografie

Pneumologie 2021; 75: 187-190

DOI 10.1055/a-1320-8250

ISSN $0934-8387$

(c) 2020. Thieme. All rights reserved.

Georg Thieme Verlag KG, Rüdigerstraße 14,

70469 Stuttgart, Germany

Korrespondenzadresse

Prof. Dr. med. Kaid Darwiche, Deutsche Gesellschaft für

Pneumologie, Sprecher der Sektion 2 „Endoskopie“, Pneumologische Universitätsklinik, Ruhrlandklinik Universitätsmedizin Essen, Tüschener Weg 40,

45239 Essen, Deutschland

kaid.darwiche@rlk.uk-essen.de

\section{ZUSAMMENFASSUNG}

Die Pandemie ausgelöst durch SARS-CoV-19 geht weltweit unverändert mit einer bedeutsamen Morbidität und Mortalität einher, sodass Schutzmaßnahmen zur Verhinderung der Transmission des Virus weiterhin notwendig sind. Mitarbeiter im Gesundheitswesen sind einem höheren Risiko ausgesetzt, und dies gilt insbesondere im Rahmen sog. Aerosol-generierender Verfahren wie der Bronchoskopie. Seit der Veröffentlichung konsentierter Empfehlungen zur Durchführung einer Bronchoskopie unter diesen Bedingungen vor mehr als einem halben Jahr hat sich die Gefahrenlage nicht wesentlich verändert, aber aufgrund des erheblichen Erkenntnisgewinns in der Zwischenzeit war ein Update der Empfehlungen notwendig. Die erneuerten Empfehlungen umfassen u.a. die Verminderung der Aerosolentstehung, den persönlichen Schutz der beteiligten Personen sowie Maßnahmen zur besseren Organisation der Abläufe in der Endoskopie, um eine sichere Durchführung auch in Zeiten von COVID-19 zu gewährleisten.

\section{ABSTRACT}

The SARS-CoV-19 pandemic continues to be globally related with significant morbidity and mortality, making protective measures to prevent transmission of the virus still necessary. Healthcare employees are exposed to a higher 
risk of infection and this is particularly true when performing aerosol-generating procedures such as bronchoscopy.

Since the publication of recommendations for performing a bronchoscopy in the times of COVID-19 more than six months ago, the risk situation has not changed significantly, but due to the considerable gain in knowledge in the meantime, an update of the recommendations was necessary.
The updated recommendations include the reduction of aerosol formation, the personal protection of the people involved in the procedure, as well as measures to better organize the processes in the endoscopy suite in order to perform bronchoscopic procedures securely even in times of COVID-19.
Das neuartige Coronavirus SARS-CoV-2 führt seit Anfang Dezember 2019, beginnend in der chinesischen Stadt Wuhan, zu einer bisher steigenden Anzahl an Infektionen weltweit, genannt COVID-19. Betroffene Patienten klagen am häufigsten über Fieber und Husten, bei schweren Verläufen auch über Dyspnoe. Gastrointestinale Symptome können selten zusätzlich auftreten. Geruchs- und Geschmacksstörungen sind ein typisches und häufiges Symptom [1, 2]. 80 \% der COVID-19-Infektionen verlaufen mild, intensivpflichtige Verläufe treten in $5 \%$ auf [3]. Im Gesundheitswesen tätige Personen machen bis zu 1/3 der an COVID-19 erkrankten Patienten aus [4]. Mittlerweile hat das SARS-CoV-2 zu einer weltweiten Pandemie mit mehr als 40 Millionen infizierten Patienten und fast 1,2 Millionen Toten geführt [5].

Auch in Deutschland steigen die Infektionszahlen rasant, immer mehr Landkreise übersteigen die 7-Tage-Inzidenz von mehr als 100 Neuinfektionen/100000 Einwohner [6]. Somit ist auch von einer zunehmenden (asymptomatischen) Durchseuchung in der Bevölkerung auszugehen, sodass eine Überarbeitung unserer Empfehlungen zur Durchführung einer Bronchoskopie in Zeiten der COVID-19-Pandemie notwendig wurde [7].

Der wesentliche Übertragungsweg von SARS-CoV-2 erfolgt als Tröpfcheninfektion, sodass COVID-19-Erkrankte oder -Verdächtige neben Schutzkitteln, Handschuhen und Schutzbrillen nur mit FFP-Masken betreut werden sollten. Bei rasch ansteigenden Infektionszahlen können die Infektionsketten nicht mehr sicher identifiziert und unterbrochen werden, sodass insbesondere milde Verlaufsformen, die $80 \%$ der Fälle ausmachen, nicht immer sicher identifiziert werden können. Auf der anderen Seite gehört die Bronchoskopie zu den Prozeduren, die im besonderen Maße zu einer ausgeprägten Aerosolbildung führen und somit ein hohes Infektionsrisiko bedeuten. Nach Vorgaben des „Center for Disease Control and Prevention“ sollten im Falle eines Mangels an Schutzmasken diese prioritär für Prozeduren mit ausgeprägter Aerosolbildung wie Bronchoskopien eingesetzt werden [8].

Diese Vorgaben dienen dazu, bronchoskopisch tätigen Ärzten Handlungsempfehlungen an die Hand zu geben, um in der aktuellen COVID-19-Pandemie betroffene und nicht betroffene Patienten, aber auch das Personal zu schützen, das Risiko von Übertragungen zu minimieren sowie die Aufrechterhaltung der klinischen Versorgung aller Patienten und die Verfügbarkeit von persönlichen Schutzausrüstungen sicherzustellen.

\section{Generelle Empfehlungen zu Schutz-}

\section{maßnahmen}

- Der lokalen Infektionsrate und den Krankenhauskapazitäten angepasst, strenge Indikationsstellung zur Durchführung von Bronchoskopien. Die Dringlichkeit des Eingriffs wird orientierend in $>$ Tab. 1 dargestellt.

- Bei planbaren bronchoskopischen Eingriffen sollte eine negative PCR-Testung auf SARS-CoV-2 (nicht älter als 48 Stunden) zum Eingriff vorliegen.

- Der COVID-19-Schnelltest (AG-POCT) ersetzt aufgrund einer geringeren Sensitivität nicht die SARS-CoV-2-PCR-Testung und kann insbesondere bei asymptomatischen Patienten eine SARS-CoV-2-Infektion nicht mit absoluter Sicherheit ausschließen. Die Sensitivität zur Detektion hochinfektiöser Patienten ist aber ausreichend, sodass bei dringlicher Indikation und fehlendem PCR-Ergebnis entweder ein „NotfallPCR-Test“ oder, falls nicht möglich, ein COVID-19-Schnelltest durchgeführt werden kann $[9,10]$.

- Falls bei lebensbedrohlichen Situationen das Testergebnis nicht abgewartet werden kann, sollte der Patient wie bei gesichertem Nachweis von SARS-CoV-2 untersucht werden, insbesondere mit FFP3-Maske.

- Reduktion der Mitarbeiter (Bronchoskopierende, Bronchoskopieassistenz, ggf. Anästhesieteam), die im Bronchoskopietagesprogramm eingesetzt werden, auf ein Kernteam mit Fokussierung auf besonders erfahrene Untersucher.

- Besucher oder Hospitierende sollten im Untersuchungsraum vermieden werden. Einzelne Studierende oder Auszubildende können unter Beachtung der allgemeinen Hygiene-

- Tab. 1 Einteilung der Dringlichkeit einer Bronchoskopie während der SARS-CoV2-Pandemie.

\begin{tabular}{|c|c|}
\hline $\begin{array}{l}\text { Dringliche } \\
\text { Indikation }\end{array}$ & $\begin{array}{l}\text { - Zentrale Atemwegsstenose } \\
\text { - Fremdkörperaspiration } \\
\text { - Stärkere Hämoptysen }\end{array}$ \\
\hline $\begin{array}{l}\text { Intermediäre } \\
\text { Dringlichkeit }\end{array}$ & $\begin{array}{l}\text { - Diagnostische Sicherung thorakaler Tumore } \\
\text { - Stadiierung bei thorakalem Tumor } \\
\text { - Malignomsuspekte oder symptomatische } \\
\text { mediastinale/hiläre Lymphadenopathie } \\
\text { - Unklare Lungeninfiltrate }\end{array}$ \\
\hline $\begin{array}{l}\text { Elektive } \\
\text { Indikationen }\end{array}$ & $\begin{array}{l}\text { - Asymptomatische Verlaufskontrollen } \\
\text { - Interventionelle Therapie chronisch obstruktiver } \\
\text { Atemwegserkrankungen inklusive Emphysem- } \\
\text { therapie } \\
\text { - Abklärung persistierenden Hustens }\end{array}$ \\
\hline
\end{tabular}


richtlinien und der örtlichen Infektionszahlen zugelassen werden, um dem Lehrauftrag gerecht zu werden.

- Patientenbezogene Nutzung eines Einmalschutzkittels, von Einmalhandschuhen und einer FFP2-Maske ohne Ausatemventil aller Anwesenden im Untersuchungsraum.

- Berücksichtigung der RKI-Richtlinie zum An- und Ablegen der Schutzmaske [11].

- Nutzung einer personenbezogenen Einmalschutzbrille oder wiederverwendbarem Schutzvisier bei allen Anwesenden im Untersuchungsraum.

- Je nach Versorgungslage der Produkte zur persönlichen Schutzausrichtung kann in Absprache mit der jeweiligen Krankenhaushygiene eine abweichende Regelung getroffen werden. Dies kann die patientenübergreifende Nutzung von Schutzmasken oder Schutzbrillen mit Anweisungen zur Aufbereitung und zum Umgang mit den Materialien nach den Eingriffen beinhalten.

- In Gebieten mit einer erhöhten Inzidenz (z. B. 7-Tage-Inzidenz > 50/100 000) kann, in Abstimmung mit der lokalen Krankenhaushygiene und der jeweiligen Gesundheitsbehörde, erwogen werden, in Abhängigkeit vom jeweiligen Testkonzept der Einrichtung, Mitarbeiter, die vermehrt COVID-19-Patienten betreuen, regelmäßig zu testen. Werden hierzu Antigentests verwendet, sollte ein Test mit einer sehr hohen Spezifität (>98\%) zum Einsatz kommen und eine obligate Bestätigung positiver Ergebnisse per PCR.

\section{Vermeidung und Reduktion}

\section{von Aerosolbildung}

- Starre Bronchoskopie mit JET-Ventilation sollte, soweit medizinisch vertretbar, durch geschlossene Beatmungssysteme (Tubus, Larynxmaske) ersetzt werden. Dies gilt insbesondere, wenn kein Test oder ein positiver Test auf SARS-CoV-2 vorliegt.

- Falls starre Bronchoskopie unvermeidbar, dann Nutzung mit konventioneller Beatmung und Reduktion des Austritts von Aerosol, z. B. mittels FLUVOG-Aufsatz oder sog. Mainzer Universaladapter (s. A Abb. 1).

- Bei flexiblen Bronchoskopien (ohne Nutzung eines Tubus) sollte der transnasale Zugang bevorzugt und ein zusätzlicher geschlitzter Mund-Nasen-Schutz für den Patienten genutzt werden. Alternativ kann über eine Einmalbeatmungsmaske bronchoskopiert werden und die Zu- und Abluft über ein geschlossenes Beatmungssystem sichergestellt werden (s. $>$ Abb. 2).

- Falls Untersuchungsräume mit raumlufttechnischen Anlagen der Raumluftklasse I oder II (gemäß DIN 1946-4) vorhanden sind, sollten diese bevorzugt zur Durchführung der bronchoskopischen Eingriffe genutzt werden.

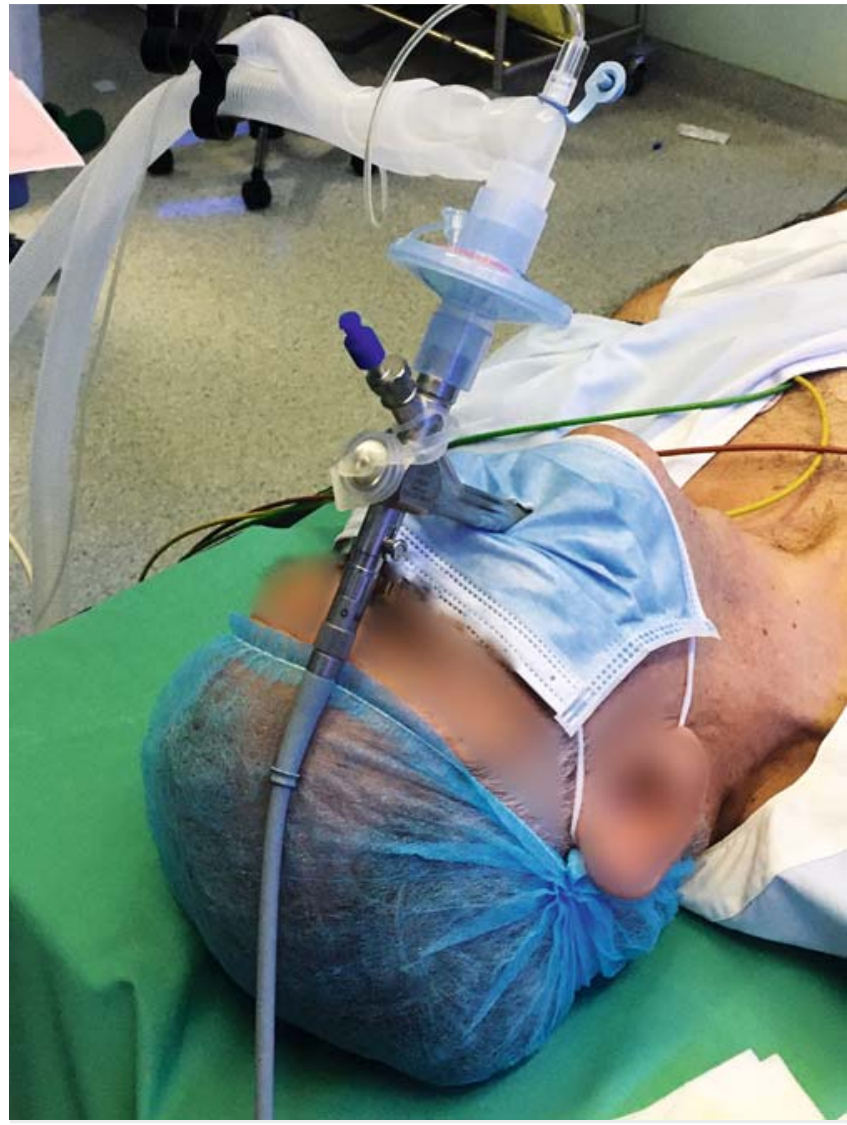

- Abb. 1 Verminderung der Aerosolbildung im Rahmen einer starren Bronchoskopie durch Nutzung eines zusätzlichen geschlitzten Mund-Nasen-Schutzes für den Patienten und Ersatz der Jet-Ventilation durch eine konventionelle Beatmung.

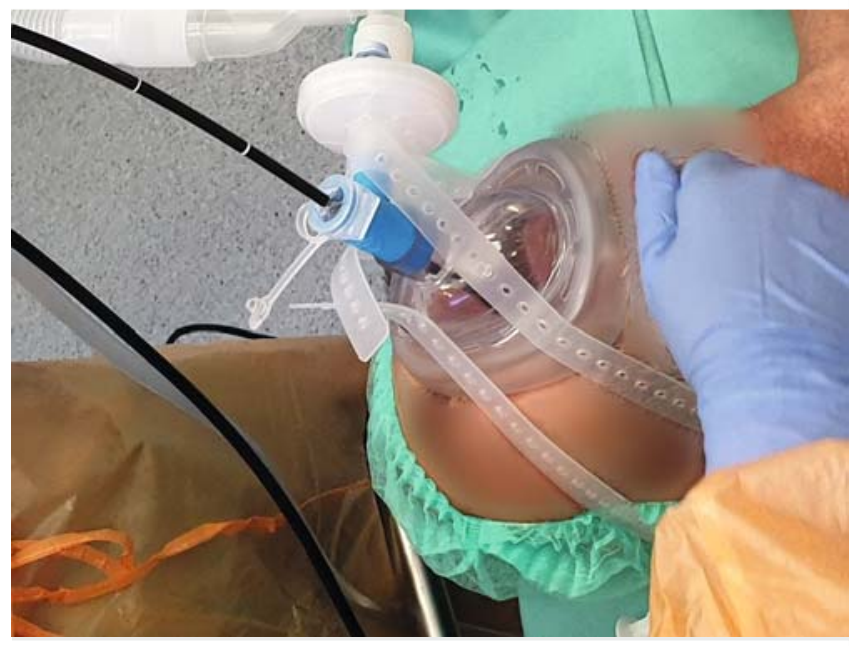

- Abb. 2 Einsatz einer Einmalbeatmungsmaske zur Aerosolreduktion bei Durchführung einer flexiblen Bronchoskopie bei Patienten mit Verdacht auf oder gesicherter SARS-CoV-2-Infektion. 


\section{Umgang bei gesicherter COVID-19- \\ Erkrankung}

- Sehr kritische Indikationsstellung zur Bronchoskopie (in Abhängigkeit der lokalen Infektionslage und der Behandlungskapazitäten im jeweiligen Krankenhaus).

- Die Indikation zur Bronchoskopie bei gesicherter oder vermuteter COVID-19-Erkrankung sollte sehr streng gestellt und nur in Betracht gezogen werden, wenn

1. der Nasopharyngealabstrich negativ ist und klinisch weiterhin eine hohe Wahrscheinlichkeit für eine SARSCoV-2-Infektion besteht,

2. andere Diagnosen in Betracht gezogen werden, die das klinische Management erheblich verändern würden oder

3. eine dringende lebensrettende Intervention notwendig ist (z. B. Haemoptoe, höhergradige benigne oder maligne zentrale Atemwegsstenose, Fremdkörperaspiration, Dilatationstracheotomie).

- Falls eine Bronchoskopie zwingend notwendig ist, sollten bei einer diagnostischen oder interventionellen Bronchoskopie FFP3-Masken genutzt werden, und die Durchführung sollte durch einen erfahrenen Untersucher erfolgen.

- Bei sichergestellter zeitnaher und validierter Aufbereitung der Bronchoskope muss der Aufbereitungsprozess zur Desinfektion der Geräte nicht geändert werden [12].

Interessenkonflikt

Die Autorinnen/Autoren geben an, dass kein Interessenkonflikt besteht.
Literatur

[1] Guan W], Ni ZY, Hu Y et al. China Medical Treatment Expert Group for Covid-19. Clinical Characteristics of Coronavirus Disease 2019 in China. N Engl J Med 2020; 382: 1708-1720

[2] Meng X, Deng Y, Dai $Z$ et al. COVID-19 and anosmia: a review based on up-to-date knowledge. Am J Otolaryngol 2020; 41: 102581102581

[3] Wu Z, McGoogan JM. Characteristics of and Important Lessons from the Coronavirus Disease 2019 (COVID-19) Outbreak in China: Summary of a Report of 72 ? 314 Cases From the Chinese Center for Disease Control and Prevention. JAMA 2020; 323: 1239-1242

[4] Wang D, Hu B, Hu C et al. Clinical Characteristics of 138 Hospitalized Patients With 2019 Novel Coronavirus-Infected Pneumonia in Wuhan, China. JAMA 2020; 323: 1061-1069

[5] https://covid19.who.int/ (Stand 2.11.2020)

[6] https://www.rki.de/DE/Content/InfAZ/N/Neuartiges_Coronavirus/ Daten/Fallzahlen_Kum_Tab.html (Stand 2.11.2020)

[7] Darwiche K, Ross B, Gesierich W et al. Empfehlungen zur Durchführung einer Bronchoskopie in Zeiten der COVID-19-Pandemie. Pneumologie 2020; 74: 260-262

[8] https://www.cdc.gov/coronavirus/2019-ncov/hcp/infection-controlrecommendations.html (abgerufen am 2.11.2020)

[9] Krüger L], Gaeddert M, Koeppel L et al. for the study team. Evaluation of the accuracy, ease of use and limit of detection of novel, rapid, antigen-detecting point-of-care diagnostics for SARS-CoV-2. medRxiv 2020. doi:10.1101/2020.10.01.20203836

[10] Porte L, Legarraga P, Vollrath V et al. Evaluation of a novel antigenbased rapid detection test for the diagnosis of SARS-CoV-2 in respiratory samples. Int J Infect Dis 2020; 99: 328-333

[11] https://www.rki.de/DE/Content/InfAZ/N/Neuartiges_Coronavirus/ PSA_Fachpersonal/Dokumente_Tab.html (abgerufen am 2.11.2020)

[12] https://www.cdc.gov/infectioncontrol/guidelines/disinfection/healthcare-equipment.html (abgerufen am 2.11.2020) 